Research Article

\title{
An Interactive Approach for Solving the Multiobjective Minimum Cost Flow Problem in the Fuzzy Environment
}

\author{
Majed G. Alharbi, ${ }^{1}$ Hamiden Abd El-Wahed Khalifa, ${ }^{2}$ and E. E. Ammar ${ }^{3}{ }^{3}$ \\ ${ }^{1}$ Department of Mathematics, College of Arts and Sciences, Methnab, Qassim University, Buridah, Saudi Arabia \\ ${ }^{2}$ Operations Research Department, Faculty of Graduate Studies for Statistical Research, Cairo University, Giza, Egypt \\ ${ }^{3}$ Mathematics Department, Faculty of Science, Tanta University, Tanta, Egypt
}

Correspondence should be addressed to E. E. Ammar; amr.saed@ymail.com

Received 17 July 2020; Revised 7 September 2020; Accepted 26 October 2020; Published 17 November 2020

Academic Editor: Basil K. Papadopoulos

Copyright (C) 2020 Majed G. Alharbi et al. This is an open access article distributed under the Creative Commons Attribution License, which permits unrestricted use, distribution, and reproduction in any medium, provided the original work is properly cited.

\begin{abstract}
This paper deals with the multiobjective minimum cost flow (F-MOMCF) with fuzzy penalty characterized by trapezoidal fuzzy numbers. Through the use of $\alpha$-cut, the F-MOMCF problem is transformed into the $\alpha$-MOMCF problem. The $\alpha$-MOMCF problem can be solved using an interactive approach combined with the weighting Tchebycheff problem. The advantages of this method are that it elicits information from the decision maker (DM) to modify the given constraint set, it gives the optimum penalty, and the effort required for obtaining the solution is reduced. The stability set of the first kind related to the $\alpha$-best compromise solution is determined. A numerical example is given for illustration and to check the validity of the approach.
\end{abstract}

\section{Introduction}

Network optimization [1, 2] makes a large part of combinatorial optimization. Pardalos et al. and Migdalas et al. [3, 4] presented a model which is used for a large number of real-world applications in numerous disciplines including communications [5], production and inventory planning, transportation, construction projects [6], water resources management [7], and supply chain management [8]. Minimum cost flow (MCF) problem is one of the most important problems in combinatorial optimization, and it is also a generalized network flow problem which aims to find the least cost of the shipment of a commodity through a capacitated network so as to satisfy demands at certain nodes from available supplies at other nodes. It has enormous applications in practical problems such as transportation, communication, urban design, and job scheduling models $[9,10]$. In addition, it is used for solving several real-world problems such as nurse scheduling, project assignment, college course assignment, and multistage production inventory (Ahuja et al.
[11]). In real-life situations, fuzzy parameters may be involved since parameters are not always constant.

Fuzzy set theory introduced by Dubois and Prade [12] has been widely used to solve many practical problems, including financial risk management, since it allows us to describe and treat imprecise and uncertain elements present in a decision problem. Then, the imperfect knowledge of the returns on the assets and the uncertainty involved in the behavior of financial markets may also be introduced by means of fuzzy $m$ quantities and/or fuzzy constraints. Kumar et al. [13] extended the use of algebraic operations on real numbers to fuzzy numbers by the use of a fuzzification principle. Sakawa and Yano [14] proposed a new computing procedure for solving fuzzy Pythagorean TP, where they extended the interval basic feasible solution and then the existing optimality method to obtain the cost of transportation. Orlovski [15] introduced the concept of $\alpha$-Pareto optimality of fuzzy parametric programs. One of the difficulties which occurs in the application of mathematical programming is that the parameters in the problem formulation are not constants but fluctuating and uncertain. Tanaka and Asai [16] formulated general multiobjective 
nonlinear programming problems with fuzzy parameters. El-Sherbeny [17] formulated multiobjective linear programming problems with fuzzy parameters. Ghatee and Hashemi [18] developed a new algorithm of the fuzzy minimum cost flow problem with fuzzy time windows (FMCFPFTW) with an efficient polynomial-time algorithm based basically on Dijkstra's algorithm. Kumar and Kaur [19] defined the total order on L-R fuzzy numbers and introduced three models for the MCF problem: MCF problem with fuzzy cost, MCF problem with fuzzy supplydemand, and a combination of two cases. Hamacher et al. [20] proposed a new method for solving the single- and multiobjective MCF problem in the fuzzy environment. Sifaleras [21] reviewed theory and algorithms for solving the multiobjective MCF problem that is for continuous and integer case exact and approximation algorithms. Shilaja and Ravi [22] gave a survey of minimum cost network flow (MCNFP). Chen et al. [23] developed an enhanced flower pollination algorithm to solve the optimal power flow (OPF) problem with multiple objectives of minimizing generating cost, transmission loss, and power plant emission and to improve voltage stability. Pankajakshan et al. [24] proposed a novel constraint processing approach, namely, the constraints-prior Pareto-domination approach, for solving the constrained multiobjective optimal power flow problem. Osman [25] proposed a multiobjective optimal experimental design framework to enhance the efficiency of the online model-identification platform.

In his earlier work, Osman and El-Banna [26] analyzed the concepts of the solvability set and the stability set of the first and second kinds for parametric convex nonlinear programming problems. Hu et al. [27] introduced the stability of fuzzy multiobjective nonlinear programming problems. Hu and Lee [28] presented a method for the MCF problem which holds complementary slackness and found an augmenting path with the dual approach.

In this paper, the minimization problem with fuzzy objective function parameters is reduced into an intervalvalued problem and hence into the multiobjective problem with the upper bound and the center of the objective function interval. The objective of the problem is considered as the minimization of the worst and the average case, respectively. The solution sets of our interval optimization problems are the efficient solutions of the corresponding multiobjective problem. Therefore, the optimal solutions against the worst and the average case are defined.

The paper is organized as follows: in Section 2, the multiobjective minimum cost flow problem in the fuzzy environment is formulated. Section 3 proposes the solution procedure for obtaining optimal compromise solution, and the parametric study related to the $\alpha$-best compromise solution is defined and determined. In Section 4, an example is given for illustration. Finally, some conclusions are reported in Section 5.

\section{Problem Formulation and Solution Concepts}

Consider the following fuzzy multiobjective minimum cost flow (F-MOMCF) problem [29]:

$$
\begin{aligned}
& (F-\mathrm{MOMCF}) \min \tilde{f}_{r}(\tilde{x}, c) \sum_{(i, j) \in Q} \widetilde{c}_{i j}^{r} x_{i j}, r=1,2, \ldots, k, \\
& \text { subject to } X= \begin{cases}\sum_{j:(i, j) \in Q} x_{i j}-\sum_{k:(k, i) \in Q} x_{l i}=b(i), & \text { for all } i \in V, \\
x_{i j} \in U_{i j}, \text { for all }(i, j) \in Q x_{i j} \geq 0, & \text { for all }(i, j) \in Q,\end{cases}
\end{aligned}
$$

where $Q$ is the set of $\operatorname{arcs}(i, j), V$ is the set of nodes, $x_{i j}$ is the decision variable representing the flow through $\operatorname{arc}(i, j), U$ is the capacity of $\operatorname{arc}(i, j), \widetilde{c}_{i j}^{r}$ is the fuzzy penalty per unit of flow through arc $(i, j)$ in the $\widetilde{c}_{i j}^{r}$ objective function, $r=1,2, \ldots, k$, and $b(i)$ is the net flow generated at node $i$, the values of $b(i)$ being positive, zero, or negative which classify node $i$ as a supply node, transshipment node, or demand node, respectively.

It is noted that the parameters $c_{i j}^{r}$ are vectors of fuzzy numbers [12]. The fuzzy numbers $\widetilde{c}_{i j}^{r}$ form a convex continuous fuzzy subset of $R$ whose membership functions $\mu_{c_{i j}}^{r}\left(c_{i j}^{r}\right)$ are defined by

(1) A continuous mapping from $R$ to the closed interval $[0,1]$

(2) $\mu_{\tilde{p}}(p)=0$ for all $\left.\left.p \in\right]-\infty ; p_{1}\right]$

(3) Strict increase on $\mathrm{p} \in] p_{1}, p_{2}[$
(4) $\mu_{\tilde{p}}(p)=1$ for all $\mathrm{p} \in\left[p_{2}, p_{3}\right]$

(5) Strict decrease on $\mathrm{p} \in] p_{3}, p_{4}[$

(6) $\mu_{p}(p)=0$ for all $p \in\left[p_{4}, \infty[\right.$

Figure 1 illustrates the graph of the membership function of a fuzzy number $\widetilde{p}$.

In this paper, assume that the F-MOMCF problem is stable [30].

Definition 1 (see [12]). The $\alpha$-level set of fuzzy numbers $c_{i j}^{r}$ is defined as the ordinary set

$$
L_{\alpha}\left(c_{i j}^{r}\right)=\left\{c_{i j}^{r}: \mu_{i j}^{r}\left(c_{i j}^{r}\right) \geq \alpha, r=1,2, \ldots, k ; \quad(i, j) \in Q\right\} .
$$

For a certain $\alpha$, the F-MOMCF problem becomes [15] 


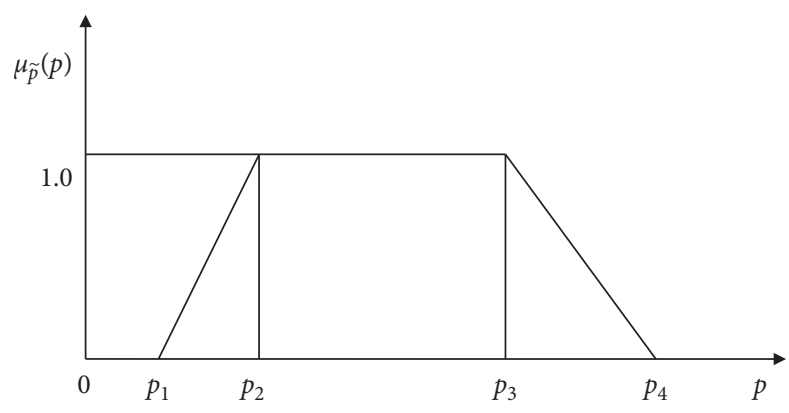

Figure 1: Representation of the membership function $\mu_{\mathrm{pp}}$ [12].

$$
\begin{aligned}
&(\alpha-\text { MOMECF }) \min f_{r}(x, c) \sum_{(i, j) \in Q} c_{i j}^{r} x_{i j}, r=1,2, \ldots, k, \\
& \text { subject to } \quad X=\left\{\begin{array}{l}
\sum_{j:(i, j) \in Q} x_{i j}-\sum_{k:(k, i) \in Q} x_{l i}=b(i), \quad \text { for all } i \in V, \\
x_{i j} \in U_{i j}, \text { for all }(i, j) \in Q x_{i j} \geq 0,
\end{array} \text { for all }(i, j) \in Q,\right. \\
& c_{i j}^{r} \in L_{\alpha}\left(\tilde{c}_{i j}^{r}\right) .
\end{aligned}
$$

Since the F-MOMCF problem is stable, the $\alpha$-MOMCF problem is also stable. It is noted that the parameters $c_{i j}^{r}$ are treated as decision variables rather than constraints.

Definition 2 (see [14]). $x^{*} \in X$ is said to be an $\alpha$-Pareto optimal solution to the $\alpha$-MOMCF problem if and only if there does not exist another $x_{i j} \in X, \in L_{\alpha}\left(\widetilde{c}_{i j}^{r}\right)$ such that $f(x$, $c) \leq f\left(x^{*}, c^{*}\right)$ and $f(x, c) \neq f\left(x^{*}, c^{*}\right)$, where the corresponding values of parameters $c^{*}$ are called $\alpha$-level optimal parameters.

Assume that the $\alpha$-MOMCF problem is stable [30].

Definition 3. $x_{i j}^{\circ} \in X,\left(c_{i j}^{r}\right)^{\circ} \in\left(\widetilde{c}_{i j}^{r}\right)_{\alpha}$, is an $\alpha$-parametric efficient solution for the $\alpha-$ MOMCF if and only if there is no $x_{i j} \in M,\left(c_{i j}^{r}\right)^{\circ} \in\left(\widetilde{c}_{i j}^{r}\right)_{\alpha}$, such that $\sum_{(i, j) \in Q} c_{i j}^{r} x_{i j} \sum_{(i, j) \in Q}$ $\left(c_{i j}^{r}\right)^{\circ} x_{i j}^{\circ}$ and $\sum_{(i, j) \in Q} c_{i j}^{r} x_{i j}<\sum_{(i, j) \in Q}\left(c_{i j}^{r}\right)^{\circ} x_{i j}$ for at least one $\mathrm{r}(r=\overline{1, K})$, where $c_{i j}^{r}$ are the $\alpha$-level optimal parameters.

Theorem 1. A point $x^{\circ} \in M$ is $\alpha$-fuzzy efficient of F-MOMCF iff for $c_{i j}^{r^{\circ}} \in\left(\widetilde{c}_{i j}^{r}\right)_{\alpha}, \widehat{x}$ is $\alpha$-parametric efficient of $\alpha-M O M C F$.

Proof. Necessity: le $x^{*}\left(c_{i j}^{r}\right) \in X$ be a fuzzy efficient solution to the F-MOMCF problem and not an $\alpha$-parametric efficient solution of the $\alpha$-MOMCF problem; then, there exist $\bar{x}\left(c_{i j}^{r^{\circ}}\right) \in M$ for $c_{i j}^{r^{\circ}} \in\left(\widetilde{c}_{i j}^{r}\right)$ such that $\widetilde{f}_{r}\left(\bar{x}, \widetilde{c}^{r}\right) \leq \widetilde{f}_{r}\left(x^{*}, \widetilde{c}^{r}\right)$ for $r$ and $\tilde{f}_{r}\left(\bar{x}, \widetilde{c}^{r}\right) \neq \tilde{f}_{r}\left(x^{*}, \widetilde{c}^{r}\right)$ for some $r$. This leads to

$$
\mu_{(\widetilde{c})=}\left\{\begin{array}{c}
c \in \mathbb{R}^{K(m \times n)}: \tilde{f}_{1}\left(\bar{x}, \widetilde{c}^{r^{\circ}}\right) \leq \tilde{f}_{1}\left(x^{\circ}, \widetilde{c}^{r^{\circ}}\right), \ldots, \\
\tilde{f}_{r-1}\left(\bar{x}, \widetilde{c}^{r^{\circ}}\right) \leq \tilde{f}_{r-1}\left(x^{\circ}, \widetilde{c}^{r^{\circ}}\right), \tilde{f}_{r}\left(\bar{x}, \widetilde{c}^{r^{\circ}}\right) \leq \tilde{f}_{r}\left(x^{\circ}, \widetilde{c}^{r^{\circ}}\right), \\
\tilde{f}_{r+1}\left(\bar{x}, \widetilde{c}^{r^{\circ}}\right) \leq \tilde{f}_{r+1}\left(x^{\circ}, \widetilde{c}^{r^{\circ}}\right), \ldots, \tilde{f}_{K}\left(\bar{x}, \widetilde{c}^{r^{\circ}}\right) \leq \tilde{f}_{K}\left(x^{\circ}, \widetilde{c}^{r^{\circ}}\right)
\end{array}\right\} \geq \alpha,
$$

$\alpha \in[0,1]$ and with strict inequality holds for at least one $r$, which contradicts that $x^{\circ}\left(c^{\circ}\right) \in M$ is $\alpha$-fuzzy efficient solution to F-MOMCF; then, $x^{\circ}\left(c^{\circ}\right) \in M$ is an $\alpha$-parametric efficient solution of the $\alpha$-MOMCF problem.
Sufficiency 1. 1Let $x^{\circ}\left(c^{\circ}\right) \in M$ be an $\alpha$-parametric efficient solution of the $\alpha$-MOMCF problem but not an $\alpha$-fuzzy efficient solution to the F-MOMCF problem. Then, there exist $\overline{\bar{X}}\left(\widetilde{c}^{r^{\circ}}\right) \in X^{\prime}$ such that

$$
\mu_{(\widetilde{c})}=\left\{\begin{array}{c}
c \in \mathbb{R}^{K(m \times n)}: \tilde{f}_{1}\left(\bar{x}, \widetilde{c}^{r^{\circ}}\right) \leq \tilde{f}_{1}\left(x^{\circ}, \widetilde{c}^{r^{\circ}}\right), \ldots, \\
\tilde{f}_{r-1}\left(\bar{x}, \widetilde{c}^{\circ}\right) \leq \tilde{f}_{r-1}\left(x^{0}, \widetilde{c}^{r^{\circ}}\right), \tilde{f}_{r}\left(\bar{x}, \widetilde{c}^{\circ}\right) \leq \tilde{f}_{r}\left(x^{\circ}, \widetilde{c}^{r^{\circ}}\right), \\
\tilde{f}_{r+1}\left(\bar{x}, \widetilde{c}^{r^{\circ}}\right) \leq \tilde{f}_{r+1}\left(x^{0}, \widetilde{c}^{r^{\circ}}\right), \ldots, \tilde{f}_{K}\left(\bar{x}, \widetilde{c}^{r^{\circ}}\right) \leq \tilde{f}_{K}\left(x^{0}, \widetilde{c}^{r^{\circ}}\right)
\end{array}\right\} \geq \alpha,
$$

$\alpha \in[0,1]$, and from the continuity and convexity of the membership function, we get

$$
\begin{aligned}
& \tilde{f}_{1}\left(\bar{x}, \widetilde{c}^{r^{\circ}}\right) \leq \tilde{f}_{1}\left(x^{\circ}, \widetilde{c}^{r^{\circ}}\right), \ldots, \tilde{f}_{r-1}\left(\bar{x}, \widetilde{c}^{r^{\circ}}\right) \leq \tilde{f}_{r-1}\left(x^{\circ}, \widetilde{c}^{r^{\circ}}\right), \\
& \tilde{f}_{r}\left(\bar{x}, \widetilde{c}^{r^{\circ}}\right) \leq \tilde{f}_{r}\left(x^{\circ}, \widetilde{c}^{r^{\circ}}\right), \tilde{f}_{r+1}\left(\bar{x}, \widetilde{c}^{r^{\circ}}\right) \leq \widetilde{f}_{r+1}\left(x^{\circ}, \widetilde{c}^{r^{\circ}}\right), \ldots, \\
& \tilde{f}_{K}\left(\bar{x}, \widetilde{c}^{r^{\circ}}\right) \leq \tilde{f}_{K}\left(x^{\circ}, \widetilde{c}^{r^{\circ}}\right) \text {, for } c_{i j k}^{r p} \in\left(\widetilde{c}_{i j k}^{r p}\right)_{\alpha} \text {, }
\end{aligned}
$$

$i=\overline{1, m} ; j=\overline{1, n} ; r=\overline{1, K}$. This is a contradiction.

The $\alpha-$ MOMCF can be solved by using the weighting Tchebycheff problem:

$$
\min _{x \in X(U)} \max _{1 \leq r \leq}\left\{\gamma_{r}\left(f_{r}\left(x, c_{i j}^{r}\right)-f_{r}^{*}\right), c_{i j}^{r} \in L_{\alpha}\left(\widetilde{c}_{i j}^{r}\right)\right\},
$$

or equivalently,

$$
\min \left\{z: \gamma_{r}\left(f_{r}\left(x, c_{i j}^{r}\right)-f_{r}^{*}\right) \leq z, \quad r=1,2, \ldots, K, x \in X, c_{i j}^{r} \in L_{\alpha}\left(\widetilde{c}_{i j}^{r}\right)\right\},
$$

where $\gamma_{r} \geq 0, r=1,2, \ldots, K, \sum_{r=1}^{K} \gamma_{r}=1$, and $f_{r}^{*}, r=1,2$, $\ldots, K$, are the ideal targets.

Remark 1. The $\alpha-\mathrm{MOMCF}$ can be treated using the weighting problem, i.e., by considering the following problem: 
$\min \left\{\delta_{r} f_{r}\left(x, c_{i j}^{r}\right): x \in X, c_{i j}^{r} \in L_{\alpha}\left(\widetilde{c}_{i j}^{r}\right)\right\}, \delta_{r} \geq 0, \delta_{r} \neq 0, \quad r=1,2, \ldots, K$.

It is clear that $\left(x^{*}, c^{*}\right)$ is an $\alpha$-Pareto optimal solution of the $\alpha$-MOMCF if there exists $\delta^{*} \geq 0$ such that $\left(x^{*}, c^{*}\right)$ is the unique optimal solution of problem (9) corresponding to the $\alpha$-level.

The stability of problems (8) and (9) follows from the stability of the $\alpha-$ MOMCF problem.

Remark 2. Problem (8) can also be written as follows:

$$
\begin{array}{cl}
\min \left[f_{1}(x,\right. & \left.\left.c_{i j}^{1}\right)-f_{1}^{*}\right] \\
\text { subject to } & \gamma_{r}\left(f_{r}\left(x, c_{i j}^{r}\right)-f_{r}^{*}\right)-f_{1}\left(x, c_{i j}^{r}\right)+f_{1}^{*} \leq 0, \\
r=2,3, \ldots, K, & \\
& x \in X, c_{i j}^{r} \in L_{\alpha}\left(\widetilde{c}_{i j}^{r}\right) .
\end{array}
$$

\section{Solution Procedure}

In this section, the steps of the solution method for obtaining the $\alpha$-best compromise solution corresponding to the $\alpha$-level which has the minimum combined deviation from the ideal target $f_{r}^{*}$, where

$$
f_{r}^{*}=\min _{x \in X(U),(c, U) \in L_{\alpha}(c, U)} f_{r}\left(x, c_{i j}^{r}\right), \quad r=1,2, \ldots, K,
$$

and the steps are as follows:

Step 1: calculate the maximum and minimum of each objective function individually with respect to the given constraints for $\alpha=1$ and $\alpha=0$, respectively.

Step 2: compute the initial weights from the following relation:

$$
\gamma_{r}=\frac{\bar{f}_{r}-\underline{f}_{r}}{\sum_{r=1}^{K}\left(\bar{f}_{r}-\underline{f}_{r}\right)}
$$

where $\bar{f}_{r}$ is the individual maximum and $f_{r}$ is the individual minimum.

Step 3: the decision maker selects the initial value of $\alpha(0<\alpha<1)$.

Step 4: formulate and solve problem (8) to get $\left(x^{*}, c^{*}\right)$.
Step 5: determine the termination. When $f\left(x^{*}, c^{*}\right)$ is satisfactory to the DM, let $\left(x^{*}, c^{*}\right)$ be the final solution, and go to step 6. Else, return to step 3 .

Step 6: determine $S\left(x^{*}, c^{*}\right)$ as follows.

Let us consider problem (8) as in the following equivalent form:

$$
\begin{aligned}
& \min \left\{z: \gamma_{r}\left(f_{r}\left(x, c_{i j}^{r}\right)-f_{r}^{*}\right) \leq z,\right. \\
& \left.r=1,2, \ldots, K, x \in X, d_{i j}^{1 r} \leq c_{i j}^{r} \leq d_{i j}^{2 r}\right\},
\end{aligned}
$$

where $\left[d_{i j}^{1 r}, d_{i j}^{2 r}\right] \in L_{\alpha}\left(\widetilde{c}_{i j}^{r}\right),(i, j) \in Q, r=1,2, \ldots, K$. The stability of the $\alpha$-MOMCF problem implies the stability of problem (13). $S\left(x^{*}, c^{*}\right)$ can be determined by applying the following conditions:

$$
\begin{aligned}
& \eta^{r}\left(c_{i j}^{r *}-d_{i j}^{2 r}\right)=0, \quad r=1,2, \ldots, K, \\
& \zeta^{r}\left(d_{i j}^{1 r}-c_{i j}^{r *}\right)=0, \quad r=1,2, \ldots, K, \\
& \eta^{r}, \zeta^{r} \geq 0, \quad r=1,2, \ldots, K .
\end{aligned}
$$

Consider the following three cases.

Case 1: $\eta^{r}>0, r \in I_{1} \subset\{1,2, \ldots, K\} ; \eta^{r}=0, r \notin I_{1}$. $\zeta^{r}>0 . r \in I_{2} \subset\{1,2, \ldots, K\} ; \zeta^{r}=0, r \notin I_{2}$.

Let $M$ be the set of all proper subsets of $\{1,2, \ldots, K\}$. Then, $S_{I_{1}, I_{2}}\left(x^{*}, c^{*}\right)=$ $\left\{\begin{array}{c}\left(d^{1}, d^{2}\right) \in \mathbb{R}^{2 K}: d_{i j}^{2 r}=c_{i j}^{r *}, r \in I_{1}, d_{i j}^{2 r} \geq c_{i j}^{r *}, r \notin I_{1}, \\ d_{i j}^{1 r}=c_{i j}^{r *}, r \in I_{2}, d_{i j}^{1 r} \leq c_{i j}^{r *}, r \notin I_{2}\end{array}\right\}$. Hence,

$$
S_{1}\left(x^{*}, c^{*}\right)=\bigcup_{I_{1}, I_{2}} S_{I_{1}, I_{2}}\left(x^{*}, c^{*}\right) .
$$

Case $2 \eta^{r} ; \zeta^{r}=0$. Then,

$S_{2}\left(x^{*}, c^{*}\right)=\left\{\begin{array}{c}\left(d^{1}, d^{2}\right) \in \mathbb{R}^{2 K}: d_{i j}^{2 r} \geq c_{i j}^{r *}, \quad r=1,2, \ldots, K, \\ d_{i j}^{1 r} \leq c_{i j}^{r *}, \quad r=1,2, \ldots, K\end{array}\right\}$.

Case $3 \eta^{r} ; \zeta^{r}>0$. Then,

$S_{3}\left(x^{*}, c^{*}\right)=\left\{\begin{array}{c}\left(d^{1}, d^{2}\right) \in \mathbb{R}^{2 K}: d_{i j}^{2 r}=c_{i j}^{r *}, \quad r=1,2, \ldots, K, \\ d_{i j}^{1 r}=c_{i j}^{r *}, \quad r=1,2, \ldots, K\end{array}\right\}$. 
Thus,

$$
S\left(x^{*}, c^{*}\right)=\bigcup_{p=1}^{3} S_{p}\left(x^{*}, c^{*}\right)
$$

\section{Numerical Example}

Consider the following F-MOMCF problem with 5 nodes and 7 arcs as in Figure 2.

$$
\begin{aligned}
& \min \tilde{f}_{1}= {\left[(1,2,3,5) x_{12} \oplus(3,4,5,6) x_{13} \oplus(5,7,8,13) x_{24} \oplus(9,10,15,16) x_{25} \oplus(7,8,10,11) x_{34} \oplus(9,10,12,14) x_{35} \oplus(2,4,6,8) x_{45}\right], } \\
& \min \tilde{f}_{2}= {\left[(7,8,9,10) x_{12} \oplus(2,3,4,6) x_{13} \oplus(1,2,3,4) x_{24} \oplus(1,2,3,5) x_{25} \oplus(6,7,8,11) x_{34} \oplus(4,6,9,12) x_{35} \oplus(3,4,6,7) x_{45}\right], } \\
& \text { subject to } \quad x \in X=\left(\begin{array}{c}
x_{12}+x_{13}=10, x_{24}+x_{25}-x_{12}=0, \\
x_{34}+x_{35}-x_{13}=20, x_{34}+x_{35}-x_{13}=20, \\
x_{45}-x_{24}-x_{34}=-15,-x_{25}-x_{35}-x_{45}=-15, \\
0 \leq x_{12} \leq 10,0 \leq x_{13} \leq 10,0 \leq x_{24} \leq 15, \\
0 \leq x_{25} \leq 10,0 \leq x_{34} \leq 5,0 \leq x_{35} \leq 15, \\
0 \leq x_{35} \leq 15,0 \leq x_{45} \leq 10
\end{array}\right) .
\end{aligned}
$$

Step 1: solve the problems

$$
\begin{aligned}
& \min \left(x_{12}+3 x_{13}+5 x_{24}+9 x_{25}+7 x_{34}+9 x_{35}+2 x_{45}\right) \\
& \min \left(7 x_{12}+2 x_{13}+x_{24}+x_{25}+6 x_{34}+4 x_{35}+3 x_{45}\right), \\
& \max \left(5 x_{12}+6 x_{13}+13 x_{24}+16 x_{25}+11 x_{34}+14 x_{35}+8 x_{45}\right), \\
& \max \left(10 x_{12}+6 x_{13}+4 x_{24}+5 x_{25}+11 x_{34}+12 x_{35}+7 x_{45}\right),
\end{aligned}
$$$$
\text { subject to } x \in X \text {. }
$$

The individual minimum is $f_{1}=230$ and $f_{2}=170$. The individual maximum is $\overline{f_{1}}=445$ and $\overline{f_{2}}=375$. Also, the ideal targets are $f_{1}^{*}=410$ and $f_{2}^{*}=190$. Step 2: the weights

$$
\begin{aligned}
& \gamma_{1}=\frac{\bar{f}_{1}-\underline{f}_{1}}{\left(\bar{f}_{1}-\underline{f}_{1}\right)+\left(\bar{f}_{2}-\underline{f}_{2}\right)}=\frac{215}{420}=0.511905, \\
& \gamma_{2}=\frac{\bar{f}_{2}-\underline{f}_{2}}{\left(\bar{f}_{1}-\underline{f}_{1}\right)+\left(\bar{f}_{2}-\underline{f}_{2}\right)}=\frac{205}{420}=0.488095 .
\end{aligned}
$$

Step 3: suppose that the decision maker selects $\alpha=0.80$. Step 4: the subproblem to be solved is $\min z$,

$$
\begin{aligned}
& \text { subject to }\left(2.6 x_{12}+4.5 x_{13}+7.8 x_{24}+12.5 x_{25}+9 x_{34}\right. \\
& \left.\quad+11.1 x_{35}+5 x_{45}-1.9535 z\right) \leq 230, \\
& \left(8.5 x_{12}+3.6 x_{13}+2.5 x_{24}+2.6 x_{25}+7.7 x_{34}+7.6 x_{35}\right. \\
& \left.\quad+4.9 x_{45}-2.0488 z\right) \leq 170, \\
& x \in X .
\end{aligned}
$$

The $\alpha$-best compromise solution for $z=45.1484$ is $x_{12}^{*}=10, x_{24}^{*}=10, x_{34}^{*}=5, x_{35}^{*}=\underset{\widetilde{f}}{15}, x_{13}^{*}=x_{25}^{*}=x_{45}^{*}=0, f_{1}$ $=315.5$, and $f_{2}=262.5$. Also, $\widetilde{f}_{1}=(230,280,340,445)$, $\widetilde{f}_{2}=(170,225,295,375)$.

Assume that the solution is satisfactory for the DM, and hence, $S\left(x^{*}, c^{*}\right)$ is determined as

$$
\begin{aligned}
\eta^{1}\left(c_{i j}^{r *}-d_{i j}^{21}\right) & =0, r=1,2, \\
\eta^{1}\left(d_{i j}^{1 r}-c_{i j}^{r *}\right) & =0, r=1,2, \\
\eta^{1}, \eta^{2} & \geq 0 .
\end{aligned}
$$

We have $I_{1} \subseteq\{1,2\}$. For $I_{1}=\varnothing, \eta^{1}, \eta^{2}=0$. Then,

$$
S_{I_{1}}\left(x^{*}, c^{*}\right)=\left\{\begin{array}{c}
d^{2} \in \mathbb{R}^{2}: d_{12}^{21} \geq 2.6 d_{24}^{21} \geq 7.8, d_{34}^{21} \geq 9, d_{35}^{21} \geq 11.1, \\
d_{12}^{22} \geq 8.5, d_{24}^{22} \geq 2.5, d_{34}^{22} \geq 7.7, d_{35}^{22} \geq 7.6
\end{array}\right\} .
$$




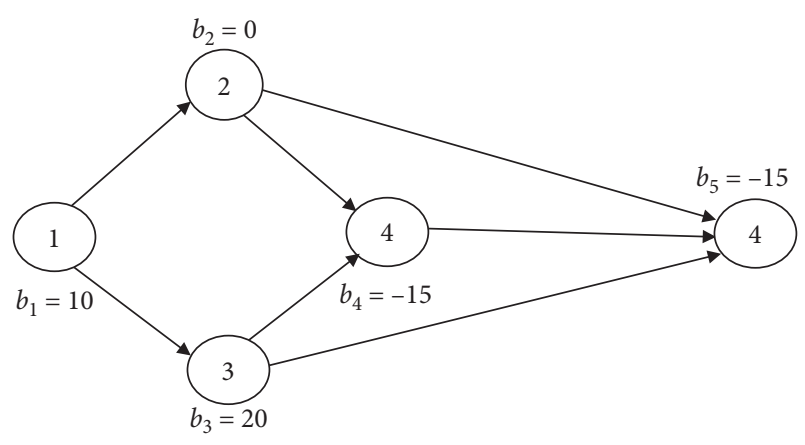

FIgURE 2: A network with 5 nodes and 7 arcs [19].

For $I_{2}=\{1\}, \eta^{1}>0$ and $\eta^{2}=0$. Then,

$S_{I_{2}}\left(x^{*}, c^{*}\right)=\left\{\begin{array}{c}d^{2} \in \mathbb{R}^{2}: d_{12}^{21}=2.6, d_{24}^{21}=7.8, d_{34}^{21}=9, d_{35}^{21}=11.1, \\ d_{12}^{22} \geq 8.5, d_{24}^{22} \geq 2.5, d_{34}^{22} \geq 7.7, d_{35}^{22} \geq 7.6\end{array}\right\}$.

For $I_{3}=\{2\}, \eta^{1}=0$ and $\eta^{2}>0$. Then,

$S_{I_{3}}\left(x^{*}, c^{*}\right)=\left\{\begin{array}{c}d^{2} \in \mathbb{R}^{2}: d_{12}^{21} \geq 2.6, d_{24}^{21} \geq 7.8, d_{34}^{21} \geq 9, d_{35}^{21} \geq 11.1, \\ d_{12}^{22}=8.5, d_{24}^{22}=2.5, d_{34}^{22}=7.7, d_{35}^{22}=7.6\end{array}\right\}$.

For $I_{4}=\{1,2\}, \eta^{1}>0$ and $\eta^{2}>0$. Then,

$S_{I_{4}}\left(x^{*}, c^{*}\right)=\left\{\begin{array}{c}d^{2} \in \mathbb{R}^{2}: d_{12}^{21}=2.6, d_{24}^{21}=7.8, d_{34}^{21}=9, d_{35}^{21}=11.1, \\ d_{12}^{22}=8.5, d_{24}^{22}=2.5, d_{34}^{22}=7.7, d_{35}^{22}=7.6\end{array}\right\}$.

Thus,

$$
S\left(x^{*}, c^{*}\right)=\bigcup_{p=1}^{4} S_{I_{p}}\left(x^{*}, c^{*}\right)
$$

\section{Conclusions}

In this paper, we have introduced fuzzy multiobjective minimum cost flow, where the penalty is characterized by trapezoidal fuzzy numbers. After converting the problem into the corresponding crisp problem, an interactive approach combined with the weighting Tchebycheff problem is applied for solving the problem, and then, $S\left(x^{*}, c^{*}\right)$ related to the solution is determined. The advantages of the approach are that it elicits information from the decision maker to modify the given constraint set, it gives the optimum penalty, and the computational effort required for obtaining the final solution is reduced. In addition, the proposed method transforms the fuzzy problem into an interval-valued problem and hence into the multiobjective problem which is significant for using in interactive methods to make any comments by related managers and achieving the logical solutions. Also, GAMS software is applied for obtaining the solution.

For future research, there are several directions worth investigating. In this paper, the authors assumed the model parameters in the fuzzy environment. However, in real-life situations, the probabilistic nature of parameters may be considered. Also, a more comprehensive model can be built by considering the neutrosophic sets to deal with the uncertainty in model parameters. Further research can build a more sophisticated model by appropriately considering the intuitionistic fuzzy sets.

\section{Data Availability}

The data used to support the findings of this research are available from the corresponding author upon request.

\section{Disclosure}

Hamiden Abd El-Wahed Khalifa is currently at Mathematics Department, College of Science and Arts, Al-Badaya, Qassim University, Buridah, Saudi Arabia.

\section{Conflicts of Interest}

The authors declare no conflicts of interest.

\section{References}

[1] C. A. S. Oliveira and P. M. Paradalos, "Mathematical aspects of network routing optimization, 53," in Series: Springer Optimization and its ApplicationsSpringer, Berlin, Germany, 2011.

[2] B. Korte and J. Vygen, "Combinatorial optimization, theory and algorithms," in Series: Algorithms and Combinatories, Springer- Verlage, Berlin, Germany, 5th edition, 2012.

[3] P. M. Pardalos, D. Z. Du, and R. L. Graham, Handbook of Combinatorial Optimization, Springer, Berlin, Germany, 2013.

[4] A. Migdalas, A. Sifaleras, C. K. Georgiadis, J. Papathanasiou, and E. Stiakakis, "Optimization theory, decision making and operations research applications," in Mathematics \& StatisticsSpringer, New York, NY, USA, 2013.

[5] J. Xu, Y. Tu, and Z. Zeng, "A nonlinear multiobjective bilevel model for minimum cost network flow problem in a largescale construction project," Mathematical Problems in Engineering, vol. 2012, Article ID 463976, 9 pages, 2012.

[6] D. Haro, J. Paredes, A. Solera, and J. Andreu, "A model for solving the optimal water allocation problem in river basins with network flow programming when introducing nonlinearities," Water Resources Management, vol. 26, no. 14, pp. 4059-4071, 2012.

[7] R. J. I. Basten, M. C. Van Der Heijden, and J. M. J. Schutten, "Practical extensions to a minimum cost flow model for level of repair analysis," European Journal of Operational Research, vol. 211, no. 2, pp. 333-342, 2011.

[8] R. K. Ahuja and P. M. Pardalos, "Network optimization problems: algorithm, applications and convexity," in Series on Applied MathematicsWorld Scientific, Singapore, 1993.

[9] M. S. Bazaraa, M. Jarvis, and H. D. Sherali, Linear Programming and Networks Flow, John Wiley \& Sons, Hoboken, NJ, USA, 1990.

[10] L. A. Zadeh, "Fuzzy sets," Information and Control, vol. 8, no. 3, pp. 338-353, 1965.

[11] R. K. Ahuja, T. L. Magnanti, and J. B. Orlin, Network Flow: Theory, Algorithms, and Applications, Prentice- Hall, Englewood Gliffs, NJ, USA, 1993.

[12] D. Dubois and H. Prade, Fuzzy Sets and Systems; Theory and Applications, Academic Press, New York, NY, USA, 1980. 
[13] R. Kumar, S. A. Edalatpanah, S. Jha, and R. Singh, "A Pythagorean fuzzy approach to the transportation problem," Complex \& Intelligent Systems, vol. 5, no. 2, pp. 255-263, 2019.

[14] M. Sakawa and H. Yano, "An interactive fuzzy satisficing method for multiobjective nonlinear programming problems with fuzzy parameters," Fuzzy Sets and Systems, vol. 89, no. 30, pp. 221-238, 1989.

[15] S. Orlovski, "Multiobjective programming problems programming with fuzzy parameters," Control Cybernetic, vol. 13, pp. 175-183, 1984.

[16] H. Tanaka and K. Asai, "Fuzzy linear programming problems with fuzzy numbers," Fuzzy Sets and Systems, vol. 13, no. 1, pp. 1-10, 1984.

[17] N. El- Sherbeny, "Algorithm of fuzzy minimum cost flow problem with fuzzy time- windows," Global Journal of Pure and Applied Mathematics, vol. 14, no. 2, pp. 219-231, 2018.

[18] M. Ghatee and S. M. Hashemi, "Generalized minimal cost flow problem in fuzzy nature: an application in bus network planning problem," Applied Mathematical Modelling, vol. 32, no. 12, pp. 2490-2508, 2008.

[19] A. Kumar and M. Kaur, "A new method for solving single and multi-objective fuzzy minimum cost flow problems with different membership functions," Sadhana, vol. 39, no. 1, pp. 189-206, 2014.

[20] H. W. Hamacher, C. R. Pedersen, and S. Ruzika, "Multiple objective minimum cost flow problems: a review," European Journal of Operational Research, vol. 176, no. 3, pp. 1404-1422, 2007.

[21] A. Sifaleras, "Minimum cost network flows: problems, algorithms, and software," Yugoslav Journal of Operations Research, vol. 23, no. 1, pp. 3-17, 2013.

[22] C. Shilaja and K. Ravi, "Multi- objective optimal power flow problem using enhanced flower pollination algorithm," Gazi University Journal of Science, vol. 30, no. 1, pp. 79-91, 2017.

[23] G. Chen, X. Yi, Z. Zhang, and H. Lei, "Solving the multi-objective optimal power flow problem using the multi-objective firefly algorithm with a constraints-prior pareto-domination approach," Energies, vol. 11, no. 12, p. 3438, 2018.

[24] A. Pankajakshan, C. Waldron, M. Quaglio, A. Graviilidis, and F. Galvanin, "A multi- objective optimal experimental design framework for enhancing the efficiency of online modelidentification platform," Engineering, vol. 18, 2019.

[25] M. S. A. Osman, "Qualitative analysis of basic notions in parametric convex programming, I (Parameters in the constraints)," Aplikace Matematiky, vol. 22, pp. 318-322, 1977.

[26] M. S. A. Osman and A. Z. H. El- Banna, "Stability of multiobjective nonlinear programming problems with fuzzy parameters," Mathematics and Computers in Simulation, vol. 35, pp. 321-326, 1993.

[27] Y. Hu, X. Zhao, J. Liu, B. Liang, and C. Ma, "An efficient algorithm for solving minimum cost flow problem with complemretary slackness," Mathemateciacl Problems in Engineering, vol. 2020, Article ID 2439265, 16 pages, 2020.

[28] H. Shin and E. S. Lee, "Fuzzy multi-level minimum cost flow problems," Fuzzy Sets and Systems, vol. 2, pp. 159-176, 1999.

[29] R. T. Rockafellar, "Duality and stability in extremum problems involving convex functions," Pacific Journal of Mathematics, vol. 21, pp. 167-181, 1967.

[30] M. Sakawa and K. Yauchi, "An interactive fuzzy satisficing method for multiobjective nonconvex programming problems with fuzzy numbers through coevolutionary genetic algorithms," IEEE Transactions on Systems, Man and Cybernetics, Part B (Cybernetics), vol. 31, no. 3, pp. 459-467, 2001. 